\title{
Estimaciones del valor del tiempo de viaje de los visitantes del Parque Nacional del Teide*
}

\author{
Rosa Marina González \\ Ángel Simón Marrero \\ Universidad de La Laguna \\ Concepción Román \\ Universidad de Las Palmas de Gran Canaria
}

\section{Resumen}

En este trabajo se estiman, por primera vez, los valores del tiempo de viaje de los visitantes del Parque Nacional del Teide (Tenerife). Para ello, se utiliza una aproximación novedosa en la literatura de la demanda recreativa consistente en la estimación de modelos de elección discreta referidos a la elección del modo de viaje. Las estimaciones obtenidas a partir de una encuesta de preferencias reveladas muestran que los visitantes valoran de forma diferente los distintos componentes del tiempo de viaje y que los valores del tiempo varían según el sexo y la edad del visitante. Estos resultados son de gran utilidad a la hora de evaluar políticas que promuevan la utilización de modos de transporte más sostenibles para el acceso a espacios naturales.

Palabras clave: modelos de elección discreta, valor del tiempo, preferencias reveladas.

Códigos JEL: C25, D61, R4.

\begin{abstract}
In this work we estimate, for the first time, the travel time values of the visitors of the Parque Nacional del Teide (Tenerife). With this purpose, we use a novel approach in the recreative demand literature based on the estimation of discrete choice models of transport mode. The estimates obtained show that the visitors evaluate the components of the travel time differently and that the travel time values vary according to the sex and age of the visitors. These results are very useful in the assessment of policies that promote the use of more sustainable transport modes for access to natural sites
\end{abstract}

Keywords: discrete choice models, value of time, revealed preferences.

JEL classification: $C 25, D 61, R 4$.

\section{Introducción}

Los parques nacionales son espacios naturales que gozan de protección legal por parte del Estado para poder preservar su riqueza ecológica. Su origen data de finales del siglo XIX y uno de los grandes retos a los que se enfrentaron los responsables de la gestión de los parques nacionales, en sus inicios, fue la mejora de su accesibilidad,

* Los autores desean agradecer a la Fundación CajaCanarias la financiación concedida para la realización del proyecto denominado «Diseño de un plan de movilidad sostenible para los visitantes del Parque Nacional del Teide y evaluación de implantación de carriles “bici” en Tenerife», del que se extraen los datos utilizados en este trabajo. Igualmente, desean agradecer al director y técnicos del Parque Nacional del Teide por la documentación e información facilitada. 
con el fin de garantizar el uso público de dichos espacios. Sin embargo, la situación reciente ha cambiado sustancialmente. En la actualidad, estos parques se enfrentan a la necesidad de cerrar e incluso desmantelar carreteras con objeto de controlar los flujos de visitantes. Tal y como señala Manning et al. (2014), el elevado volumen de visitantes a los parques nacionales ha puesto de manifiesto que el transporte es una manifestación de la tensión entre el uso y la conservación.

El incremento observado en el número de visitantes durante las últimas décadas (Ansson, 1998, Buckley, 2000; Balmford et al., 2009) ha traído consigo una variedad de externalidades negativas derivadas, en gran parte, de los patrones de movilidad del turismo de masas. De este modo, muchos espacios naturales están sometidos a los problemas de la masificación, la contaminación, el ruido, el exceso de vehículos, la saturación de aparcamientos, y al incremento en el número de accidentes (véase, por ejemplo, los trabajos de Reeves, 2006; Manning y Dougherty, 2000; Mace et al., 2004). Estos problemas comprometen no solo la sostenibilidad del espacio, sino también la calidad de la visita.

El reconocimiento de la importancia que tienen los problemas asociados a la movilidad de los visitantes ha conducido a un aumento del interés global acerca de la necesidad de establecer sistemas de transporte más sostenibles para acceder y moverse dentro de los espacios naturales protegidos. No obstante, para evaluar cualquier política de gestión de la movilidad, susceptible de ser aplicada en estos lugares, es necesario llevar a cabo un análisis coste-beneficio. Además, para realizar este análisis es indispensable contar con un estudio de la demanda que nos permita obtener estimaciones de la valoración monetaria del tiempo de viaje de los visitantes del espacio en cuestión.

En la literatura de demanda recreativa, el enfoque más utilizado para calcular el valor del tiempo de viaje ha sido el de aproximarlo a una fracción de la tasa salarial (véase por ejemplo, Hagerty y Moeltner, 2005; Gürlük y Rehber, 2008); enfoque sustentando (Cesario, 1976) en el modelo de asignación del tiempo propuesto en Becker (1965). Sin embargo, esta aproximación no ha estado exenta de críticas (Palmquist et al., 2010) debido a que, en un viaje recreativo, la medida pertinente es el coste de oportunidad del tiempo de ocio, más que el del tiempo de trabajo. De ahí que, en este trabajo, se estimen los valores del tiempo de una forma alternativa. Específicamente, se derivan a partir del intercambio entre tiempo y dinero que revelan los visitantes cuando eligen el modo de transporte para acceder a un espacio natural; un enfoque consistente con el modelo de asignación del tiempo de DeSerpa (1971).

La metodología utilizada en este trabajo consiste en la construcción de modelos de demanda desagregados basados en la teoría de la utilidad aleatoria (Domencich y McFadden, 1975). De este modo, se estiman diversos modelos de elección discreta a partir del uso de una encuesta de preferencias reveladas llevada a cabo, en el año 2016, a una muestra de visitantes del Parque Nacional del Teide (Tenerife). Este parque es el más visitado de la Red de Parques Nacionales de España y se sitúa entre los más visitados a nivel mundial. Además, es un claro ejemplo de espacio natural sometido a la presión del turismo de masas, observándose buena parte de las 
externalidades negativas asociadas a un uso abusivo del coche por parte de los visitantes del parque.

Los resultados obtenidos en este estudio permiten cuantificar, el valor de los diversos componentes del tiempo de viaje de los visitantes del Parque Nacional del Teide. De este modo, los valores obtenidos constituyen una contribución novedosa, ya que hasta ahora no se han aportado cifras similares para este tipo de usuarios y en este contexto. Así, por ejemplo, se destaca que el tiempo de espera del autobús público es el más valorado por los visitantes, dado que es el que provoca mayor desutilidad, y que los valores del tiempo varían de acuerdo a la edad y sexo de los visitantes.

El resto del artículo se estructura de la forma siguiente. En la sección 2 se describen los datos utilizados y se analiza el perfil del visitante. El marco metodológico se presenta en la sección 3. La sección 4 describe la estrategia de modelización seguida, así como los resultados obtenidos. Finalmente, las principales conclusiones alcanzadas se presentan en la sección 5.

\section{Datos}

En esta sección se presenta, en primer lugar, las características del Parque Nacional del Teide y los patrones de movilidad de los visitantes que acceden al parque. En segundo lugar, se muestra la información recogida en la encuesta de preferencias reveladas realizada a 801 visitantes del parque durante julio de 2016 y que será utilizada para estimar los modelos de elección discreta que se presentan en la siguiente sección.

\subsection{El Parque Nacional del Teide: características del parque y patrones de movilidad de los visitantes}

El Parque Nacional del Teide (Figura 1) se encuentra en la isla de Tenerife (Islas Canarias, España). El parque se creó en 1954 en reconocimiento a su singularidad volcánica y biológica, y fue declarado Patrimonio Mundial de la Humanidad por la Unesco en el año 2007. Su principal atractivo es el complejo volcánico Teide-Pico Viejo, que alcanza 3.718 metros de altura y es el pico más alto de España. Asimismo, el parque posee un gran impacto visual debido a sus condiciones atmosféricas que generan constantes cambios de tonos y texturas sobre el paisaje y forman un impresionante mar de nubes a modo de telón de fondo sobre la montaña (UNESCO, 2007).

El Parque tiene una extensión de $190 \mathrm{~km}^{2}$, pero se encuentra rodeado del Parque Natural de la Corona Forestal, que amplía el área de protección a 466 km². Cuenta con cuatro accesos, dos desde la zona norte de la isla (La Orotava y La Laguna) y dos desde la zona sur (Vilaflor y Chío), siendo los más utilizados el de Vilaflor y La Orotava. De esta forma, es atravesado por las carreteras TF-24, TF-21 y TF-38, sumando alrededor de 50 kilómetros de recorrido. No obstante, es el tramo que discurre a través de la TF-21, de aproximadamente 22 kilómetros, el que aglutina a la mayor parte de los visitantes, debido a que en él se concentran los principales hitos del parque: teleférico, Roques de García, parador y sendero de subida al pico. 
FIGURA 1

PARQUE NACIONAL DEL TEIDE

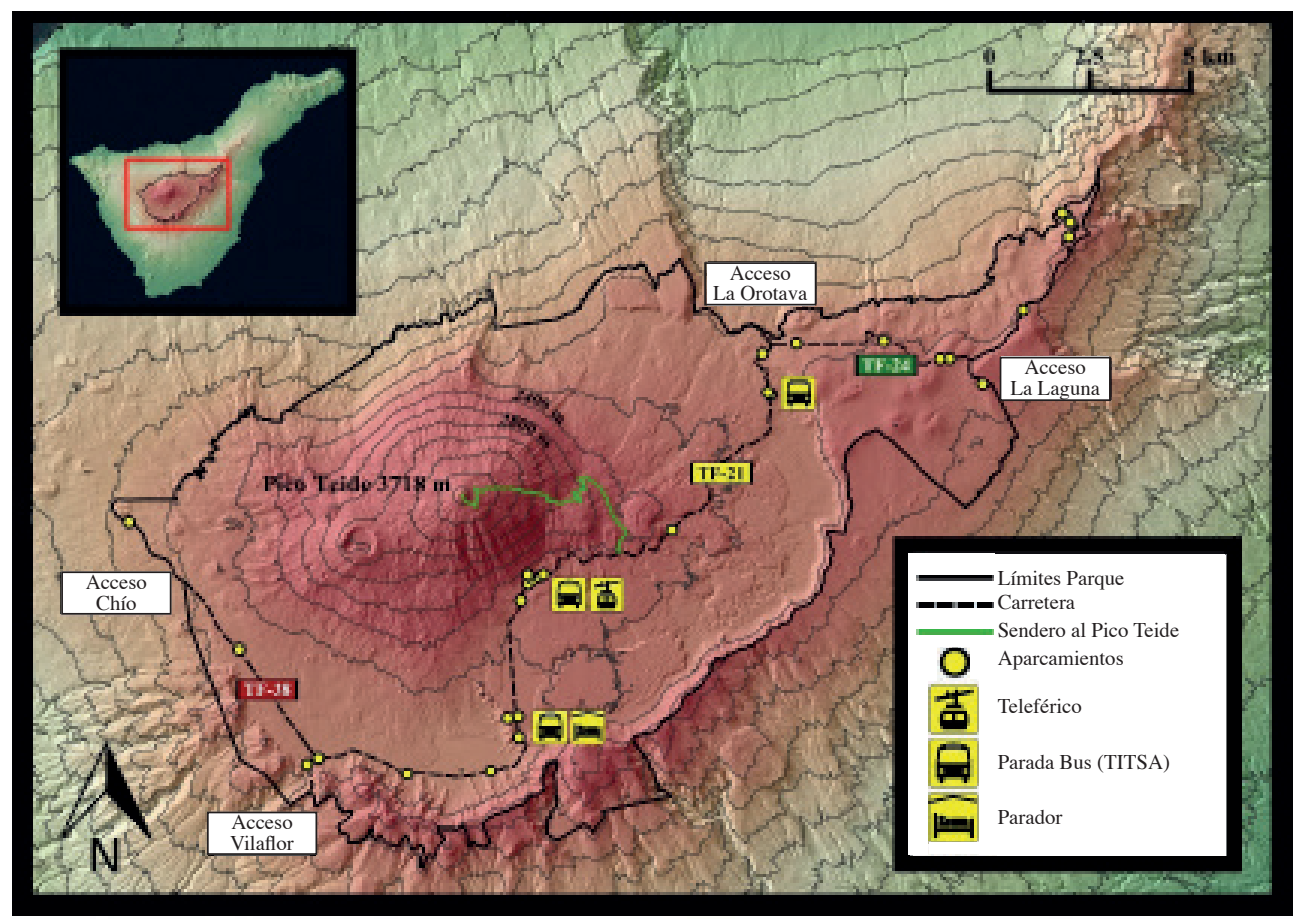

FUENTE: Elaboración propia.

Los atractivos del Parque Nacional del Teide lo han configurado como el parque nacional más visitado de España y uno de los más visitados del mundo. Durante el año 2015 recibió 3,2 millones de visitas, lo que supone un 23 por 100 del total de visitantes de los Parques Nacionales Españoles ${ }^{2}$. En el Cuadro 1 puede observarse, para el año 2015, el número de visitantes mensual, la máxima carga instantánea, las horas punta y el tiempo medio de estancia.

En el Cuadro 1 destaca, en primer lugar, un cierto comportamiento estacional en el número de visitantes mensual, siendo los meses de agosto (verano) y marzo (Semana Santa) los de máxima afluencia; y los meses de junio y julio los de mínima afluencia. Estos datos reflejan que, en promedio, el Teide recibe más de 270.000 visitantes al mes y más de 9.000 visitantes diarios. En segundo lugar, se observa que la máxima carga instantánea de visitantes, expresada como la diferencia entre las entradas y salidas para cada hora del día, supera en promedio los 2.200 visitantes. Estos visitantes tienen un tiempo medio de estancia en el parque cercano a las dos horas y se concentran mayoritariamente entre las 11 y las 12 horas de la mañana.

${ }^{2}$ Datos sobre Parques Nacionales en Anuario de Estadística, Instituto Nacional de Estadística (INE). 
CUADRO 1

VISITANTES, HORAS DE MÁXIMA AFLUENCIA Y TIEMPO MEDIO

DE ESTANCIA (2015)

\begin{tabular}{|c|c|c|c|c|c|}
\hline Meses & Visitantes & Media diaria & $\begin{array}{c}\text { Máxima } \\
\text { carga } \\
\text { instantánea }\end{array}$ & $\begin{array}{c}\text { Hora de } \\
\text { máxima } \\
\text { afluencia }\end{array}$ & $\begin{array}{c}\text { Tiempo } \\
\text { medio de } \\
\text { estancia } \\
\text { (horas) }\end{array}$ \\
\hline Enero & 275.280 & 8.880 & 2.165 & $11: 00 \mathrm{AM}$ & 1,8 \\
\hline Febrero & 264.165 & 9.434 & 2.561 & $12: 00 \mathrm{PM}$ & 1,2 \\
\hline Marzo & 336.853 & 10.866 & 2.738 & $12: 00 \mathrm{PM}$ & 1,7 \\
\hline Abril & 259.274 & 8.642 & 2.208 & $11: 00 \mathrm{AM}$ & 1,9 \\
\hline Mayo & 258.008 & 8.323 & 2.113 & $11: 00 \mathrm{AM}$ & 1,8 \\
\hline Junio & 227.821 & 7.594 & 1.856 & $11: 00 \mathrm{AM}$ & 1,7 \\
\hline Julio & 252.142 & 8.134 & 1.996 & $11: 00 \mathrm{AM}$ & 1,7 \\
\hline Agosto & 353.918 & 11.417 & 2.526 & $11: 00 \mathrm{AM}$ & 1,5 \\
\hline Septiembre & 257.325 & 8.578 & 2.094 & $11: 00 \mathrm{AM}$ & 1,7 \\
\hline Octubre & 256.300 & 8.268 & 2.182 & $11: 00 \mathrm{AM}$ & 2 \\
\hline Noviembre & 268.967 & 8.966 & 2.433 & $11: 00 \mathrm{AM}$ & 1,9 \\
\hline Diciembre & 279.390 & 9.013 & 2.461 & $11: 00 \mathrm{AM}$ & 1,8 \\
\hline Promedio & 274.120 & 9.009 & 2.278 & $11: 10 \mathrm{AM}$ & 1,7 \\
\hline
\end{tabular}

FUENTE: Informe Tragsatec para el año 2015.

El Gráfico 1 muestra la entrada de visitantes al parque y el modo de transporte utilizado para acceder según el promedio para cada día de la semana. En relación a la entrada de visitantes, se observa que los días de mayor afluencia son los del fin de semana, especialmente el domingo, con un promedio superior a 11.000 visitas. El lunes es el día en el que el parque es menos visitado, con un promedio ligeramente inferior a 8.000 visitas, no siendo una diferencia notable con el resto de días de la semana con promedios de alrededor de 9.000 visitantes/día.

En relación al modo de transporte utilizado, se aprecia que el coche es el modo de transporte predominante para acceder al Parque, con un reparto modal promedio de alrededor del 70 por 100 . El 30 por 100 restante se reparte entre los autobuses de transporte público regular y discrecional. Con respecto a los autobuses públicos, la compañía TITSA dispone de dos líneas regulares que parten desde el norte (Puerto de la Cruz) y sur (Adeje) de la isla en un único horario de mañana y se calcula que su cuota modal es de aproximadamente un 2 por $100^{3}$. Este bajo porcentaje de utilización del bus público podría deberse, entre otras causas, a la escasez de puntos en

${ }^{3}$ Esta cuota modal fue calculada a través de información proporcionada por la empresa TITSA sobre el número de viajeros en las líneas hasta el Teide para los años 2014 y 2015 y mediante un trabajo de campo, enmarcado dentro del mismo proyecto de investigación al que pertenece este trabajo, en el cual se encuestó a 372 viajeros en abril y junio de 2016 . 


\section{GRÁFICO 1}

\section{ENTRADA DE VISITANTES SEGÚN MEDIO DE TRANSPORTE. PROMEDIO SEMANAL (2015)}

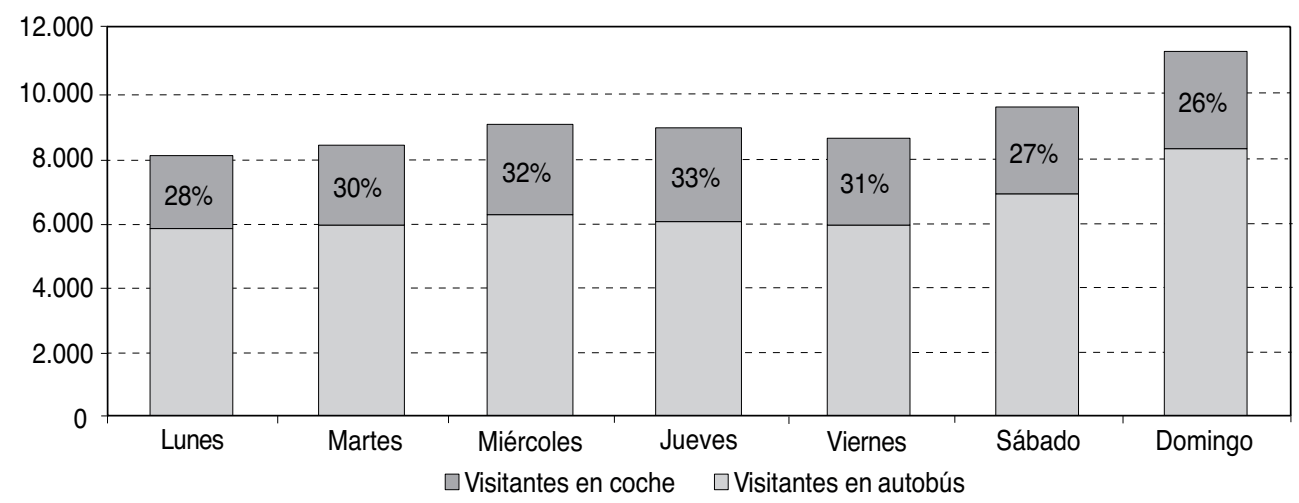

FUENTE: Informe Tragsatec para el año 2015 y elaboración propia.

la isla desde donde parten los autobuses, a la existencia de un único horario de salida (9:00) y de regreso (16:00) y a que solo se permite parar en una de las paradas disponibles en el parque (véase Figura 1), debiendo el usuario desplazarse a pie por una carretera que no dispone de aceras si quiere visitar otro lugar del parque. Ello provoca que la mayor parte de los turistas que visitan el Teide elijan el coche de alquiler como modo de acceso y, en segundo lugar, el autobús turístico para llegar al Teide.

El Cuadro 2 muestra el número de coches y autobuses que acceden al parque por meses y la máxima carga instantánea. Como es de esperar, los meses de máxima y mínima afluencia de vehículos coinciden con los meses de mayor y menor afluencia de visitantes. Para el caso de los autobuses, el promedio mensual supera los 2.200 vehículos, con un promedio diario superior a 70 y máximas cargas instantáneas de alrededor de 20 vehículos. El parque cuenta con 19 plazas de aparcamiento habilitadas para autobuses, por lo que en periodos punta se pueden producir problemas de sobreocupación. Para el caso de los coches, se observa que, en promedio, la afluencia media diaria es superior a 2.000 para todos los meses salvo para el mes de junio, alcanzándose el valor máximo en el mes de agosto, con 2.720 coches. La máxima carga instantánea alcanzó un promedio 669 coches para el año 2015, obteniéndose la cifra más elevada en el mes de febrero, con 789 vehículos dentro del parque en un determinado momento. Teniendo en cuenta que el parque cuenta con 652 plazas de aparcamiento para coches distribuidas en 33 aparcamientos, existe un claro problema de insuficiencia de plazas durante los periodos de máxima concentración de visitantes. Este problema se ve agravado en aquellos aparcamientos de mayor demanda, tales como los cercanos al teleférico (véase Figura 1), dando lugar a conflictos entre usuarios, colapsos y ocupación de arcenes y espacios protegidos. 


\section{CUADRO 2}

COCHES Y AUTOBUSES Y MÁXIMAS CARGAS INSTANTÁNEAS (2015)

\begin{tabular}{|c|c|c|c|c|c|c|}
\hline Meses & Coches & $\begin{array}{c}\text { Media } \\
\text { diaria }\end{array}$ & $\begin{array}{c}\text { Máxima } \\
\text { carga } \\
\text { instantánea }\end{array}$ & Autobuses & $\begin{array}{c}\text { Media } \\
\text { Diaria }\end{array}$ & $\begin{array}{c}\text { Máxima } \\
\text { carga } \\
\text { instantánea }\end{array}$ \\
\hline Enero & 73.631 & 2.375 & 717 & 1.981 & 64 & 12 \\
\hline Febrero & 72.465 & 2.588 & 789 & 2.001 & 71 & 19 \\
\hline Marzo & 90.309 & 2.913 & 730 & 2.625 & 85 & 27 \\
\hline Abril & 68.951 & 2.298 & 586 & 2.314 & 77 & 23 \\
\hline Mayo & 62.276 & 2.009 & 522 & 2.174 & 70 & 21 \\
\hline Junio & 54.637 & 1.821 & 471 & 2.075 & 69 & 20 \\
\hline Julio & 63.009 & 2.033 & 509 & 2.162 & 70 & 20 \\
\hline Agosto & 84.328 & 2.720 & 617 & 2.703 & 87 & 24 \\
\hline Septiembre & 69.014 & 2.300 & 583 & 2.051 & 68 & 18 \\
\hline Octubre & 69.313 & 2.236 & 608 & 2.076 & 67 & 19 \\
\hline Noviembre & 75.018 & 2.501 & 690 & 2.350 & 78 & 25 \\
\hline Diciembre & 69.714 & 2.249 & 621 & 1.924 & 62 & 18 \\
\hline Promedio & 73.526 & 2.437 & 669 & 2.219 & 73 & 20 \\
\hline
\end{tabular}

FUENTE: Informe Tragsatec para el año 2015.

\subsection{Diseño de la encuesta de preferencias reveladas y análisis de los datos}

La información utilizada en este trabajo para estimar los valores del tiempo de viaje de los visitantes del Parque Nacional del Teide proviene de una encuesta de preferencias reveladas que se diseñó para modelizar la elección del modo de transporte que utilizan los visitantes en sus desplazamientos hasta el parque ${ }^{4}$. La encuesta fue realizada en julio de 2016 a 801 visitantes del parque utilizando el método de entrevistas «cara a cara», en el que el entrevistador formula las preguntas y ayuda al encuestado a aclarar cualquier duda. Además, las encuestas fueron llevadas a cabo en tres idiomas (inglés, alemán y español) y suministradas en horario de mañana en dos de los puntos con mayor afluencia de visitantes, el parador y el teleférico.

Para obtener la muestra de encuestados se utilizó el muestreo aleatorio estratificado con estratos conformados a partir de la nacionalidad y el modo de transporte utilizado por el visitante. La muestra se estratificó en cuatro categorías de nacionalidad (15 por 100 británicos, 15 por 100 alemanes, 40 por 100 españoles y 30 por 100 resto del mundo ${ }^{5}$ ) y, a partir de la información del Gráfico 1, en tres categorías según

\footnotetext{
${ }^{4}$ Esta encuesta se realizó con la financiación obtenida en un proyecto de investigación concedido por la Fundación CajaCanarias en el año 2015.

5 Esta estratificación se realizó a partir de un estudio previo del perfil del visitante.
} 
modo de transporte (70 por 100 en coche, 28 por 100 bus turístico discrecional y 2 por 100 bus público regular).

El cuestionario diseñado incluye, en primer lugar, preguntas referidas a las características socioeconómicas: sexo, edad, lugar de residencia habitual, zona de alojamiento en la isla y motivo de la estancia en Tenerife. También se incluyeron cuestiones referidas a la visita al Teide: número de veces que había visitado el parque y número de acompañantes. Seguidamente, los encuestados debían indicar cuál es el modo de transporte que habían utilizado para llegar al parque, para lo cual tenían que elegir entre cinco modos de transporte posibles: coche de alquiler, coche privado, bus turístico, bus público y taxi. Además, en relación al modo de transporte, se les preguntó el motivo de su elección (comodidad, más barato, miedo a conducir en otro país, no dispone de carnet de conducir, excursión incluida en el paquete del viaje, otro), la hora de salida desde su alojamiento y la hora de llegada al parque, el coste, si ha tenido en cuenta otros modos de transporte alternativos y el tiempo previsto de estacionamiento si habían viajado en coche.

A partir de la muestra obtenida de 801 encuestados, hubo que hacer un tratamiento para eliminar aquellas encuestas en la que no se declaró el origen de su viaje y aquellas en las que los individuos declararon ser cautivos de un modo de transporte, es decir, solo tenían un modo de transporte disponible. Específicamente, se consideró cautivos a los encuestados que eligieron bus turístico como modo de transporte pero que ya tenían esta excursión incluida en su paquete de viaje, no realizando una elección entre modos. Por tanto, la muestra final utilizada en este trabajo está compuesta por 751 individuos y los cuatro modos de transporte más utilizados (coche de alquiler y privado y bus turístico y público) ${ }^{6}$. El Cuadro 3 muestra las principales características socioeconómicas de los encuestados y las características de su visita al parque.

Los datos del Cuadro 3 permiten detectar que en la muestra obtenida existe una distribución desigual por sexos, con una mayor proporción de visitantes hombres. Ello es debido a que la mayor parte de los conductores de los coches que acceden al parque son hombres. En cuanto a la edad, se observa que el intervalo de edad más frecuente es el de 31 a 45 años, representando prácticamente un 50 por 100 de la muestra. Nótese, también, la alta proporción de visitantes mayores a 50 años, que representan casi un 20 por 100 de los visitantes encuestados. En relación a la nacionalidad, la información obtenida refleja aproximadamente los estratos buscados durante la fase de diseño de la encuesta, con ligeras variaciones debido a la exclusión de 50 individuos de la muestra. Asimismo, la mayor parte de los visitantes (65 por 100) se alojan en el sur de la isla, que es la principal zona turística de Tenerife. Con respecto a las preguntas relacionas con la visita, los datos muestran que más del 80 por 100 de los individuos visitaban el parque por primera vez, que un 45 por 100 lo hicieron acompañados de una persona y que un 26 por 100 lo hicieron en un grupo de cuatro personas.

${ }^{6} \mathrm{El}$ modo «taxi» fue excluido debido a que representaba un porcentaje muy pequeño en el reparto modal $(0,12$ por 100$)$ e incluirlo conducía a estimaciones erróneas. 


\section{CUADRO 3}

CARACTERÍSTICAS SOCIOECONÓMICAS DE LA MUESTRA

\begin{tabular}{|l|c|l|c|}
\hline Sexo & $\mathbf{\%}$ & Veces que ha visitado el parque & \% \\
\hline Mujeres & 33,79 & Primera vez & 82,69 \\
\hline Hombres & 66,71 & Segunda vez & 9,99 \\
\hline Edad & $\mathbf{\%}$ & Más de dos veces & 4,66 \\
\hline $18-25$ años & 9,99 & No sabe/No contesta & 2,66 \\
\hline $26-30$ años & 12,38 & Número de acompañantes & \% \\
\hline $31-45$ años & 48,34 & En solitario & 1,00 \\
\hline $46-50$ años & 10,39 & 2 & 44,69 \\
\hline $51-60$ años & 14,11 & 3 & 16,73 \\
\hline 61 o más de 61 años & 4,79 & 4 & 26,22 \\
\hline Nacionalidad & $\mathbf{\%}$ & Más de 4 & 11,36 \\
\hline Alemanes & 18,77 & Zona de alojamiento & \% \\
\hline Españoles & 42,34 & Sur de la isla & 65,94 \\
\hline Británicos & 14,65 & Norte de la isla & 33,03 \\
\hline Resto del mundo & 24,23 & Resto & 1,02 \\
\hline
\end{tabular}

FUENTE: Elaboración propia.

La única fuente de información para poder evaluar la representatividad de la muestra desde un punto de vista cualitativo es la Encuesta de Turismo Receptivo realizada por el Cabildo de Tenerife ${ }^{7}$. De esta encuesta, suministrada a los turistas en el aeropuerto en el momento de su partida, se puede obtener información sobre el perfil del visitante del Parque Nacional del Teide. En ella se establece que la mayoría de los visitantes se sitúan en un intervalo de edad de entre 31 y 45 años, que el 60 por 100 visita el parque por primera vez y que un 58 por 100 realizan la visita acompañados de su pareja, una información, por tanto, cercana a la obtenida en nuestra muestra.

El Cuadro 4 muestra las elecciones y la disponibilidad de cada modo de transporte para los 751 individuos de la muestra utilizada. Sobre la disponibilidad de los modos para cada individuo, se asumió que el coche de alquiler estaba disponible para todos los encuestados salvo para aquellos que declararon como motivos de elección no tener carnet de conducir o tener miedo a conducir en otro país. La disponibilidad del bus turístico es completa para toda la muestra asumiendo que todos los individuos pueden contratar una excursión. El bus público de línea regular, por su parte, solo está disponible para aquellos visitantes que residieran u alojaran en los municipios desde donde parten estos autobuses. Por último, el modo coche privado solo está disponible para aquellos que lo eligen y que son mayoritariamente

\footnotetext{
${ }^{7}$ http://www.tenerife.es/bancodatos/
} 


\section{CUADRO 4}

\section{ELECCIONES Y DISPONIBILIDADES DE LOS MODOS DE TRANSPORTE}

\begin{tabular}{|l|c|c|c|c|}
\hline Modo de transporte & Disponibilidad & \% & Elección & \% \\
\hline Coche de alquiler & 716 & 95,34 & 559 & 75,77 \\
\hline Bus turístico & 751 & 100 & 124 & 16,51 \\
\hline Bus público & 439 & 58,46 & 16 & 2,13 \\
\hline Coche privado & 42 & 5,59 & 42 & 5,59 \\
\hline Total & & & 751 & 100 \\
\hline
\end{tabular}

FUENTE: Elaboración propia.

residentes en la isla. Con respecto a las elecciones mostradas en el Cuadro 4, nótese que los estratos por modo de transporte se alejan relativamente de las proporciones buscadas en el diseño previo debido a, como se comentó anteriormente, la eliminación de ciertos individuos de la muestra (mayoritariamente usuarios del bus turístico que eran cautivos del modo utilizado).

A pesar de la gran cantidad de datos recogidos, las encuestas no aportan información sobre las características del viaje cuando este se realizara en los modos de transporte alternativos que tiene disponible el encuestado. En estos casos, se hace necesario simular variables de nivel de servicio, básicamente tiempos de viaje y costes, con objeto de completar adecuadamente el escenario de elección. Para realizar esta tarea, se contaba con el origen del viaje o lugar de alojamiento del individuo y con el destino, por lo que los tiempos en vehículo no declarados fueron simulados mediante las herramientas que proporciona Google Maps. Para el caso del coste operativo en coche privado, se calculó utilizando la siguiente fórmula:

$$
\text { Coste }_{j}=D_{j}\left[\left(S_{g} G_{g} P_{g}\right)+\left(S_{d} G_{d} P_{d}\right)\right]
$$

Donde $_{\text {Coste }}$ es el coste del viaje en coche privado desde un determinado lugar $j$, $D_{j}$ es la distancia recorrida, $S_{g}$ y $S_{d}$ es la proporción de coches gasolina $(g)$ y diésel $(d)$ en el parque automovilístico de Tenerife (82 por 100 y 18 por 100 , respectivamente), $G_{p}$ y $G_{d}$ es la media de consumo de combustible por tipo de vehículo (10 1/100 km y $71 / 100 \mathrm{~km}$, respectivamente) y $P_{p}$ y $P_{d}$ son los precios medios anuales del combustible $(0,92 € / 1 \text { y } 0,85 € / 1 \text {, respectivamente })^{8}$.

Para el coste del coche de alquiler, al coste operativo de la ecuación [1] se le suma una cantidad media en concepto del pago del alquiler del coche y se divide por el número de acompañantes. Para el cálculo del precio medio de alquiler, se obtuvieron datos para 17 vehículos de clase b (utilitarios) y c (compactos) para los meses de julio y agosto de 2016 de las siete compañías más representativas de coches de

8 Todos estos cálculos fueron obtenidos a partir del informe Parque de vehículos por tipos, CC.AA., provincias y carburantes 2015 publicado por la DGT. 
alquiler en Tenerife. El precio medio obtenido fue de 31,6 euros/día, del cual solo se consideró un 50 por 100, ya que se asume que el Parque Nacional del Teide no es el único destino posible diario para un turista.

Para el cálculo del coste del viaje en el autobús turístico y de los tiempos de acceso y de espera en el autobús público se utilizó el método predictive mean matching (PMM), un método de imputación semiparamétrico que permite imputar valores no observados de una determinada variable continua a partir de valores observados; en nuestro caso, a partir de valores declarados por los encuestados. Sus ventajas es que los valores imputados pueden coincidir con los observados, que puede preservar relaciones no lineales y que la distribución de los valores imputados a menudo coincide estrechamente con la de los observados (Royston y White, 2011). Para el coste del viaje en el autobús público no fue necesaria la simulación ya que los precios de los billetes de cada línea fueron proporcionados por la compañía de transporte TITSA.

Por último, el Cuadro 5 presenta la información completa sobre las variables de nivel de servicio que serán utilizadas en los modelos de elección discreta estimados en la siguiente sección. Se muestran los estadísticos descriptivos asociados a las variables de tiempo de viaje y de coste para todos los modos de transporte considerados, las cuales incluyen tanto la información simulada como la declarada por los visitantes.

\section{CUADRO 5}

ESTADÍSTICOS DESCRIPTIVOS DE LAS VARIABLES DE NIVEL DE SERVICIO UTILIZADAS

\begin{tabular}{|l|c|c|c|c|}
\hline \multicolumn{1}{|c|}{ Variable } & Media & $\begin{array}{c}\text { Desviación } \\
\text { estándar }\end{array}$ & Mínimo & Máximo \\
\hline Tiempos en vehículo (en min.) & 54,87 & 5,93 & 32 & 63 \\
\hline Coche de alquiler & 92,17 & 7,69 & 39 & 120 \\
\hline Bus turístico & 96,71 & 8,66 & 60 & 120 \\
\hline Bus público & 46,72 & 4,32 & 39 & 57 \\
\hline Coche privado & \multicolumn{5}{|l}{} \\
\hline Tiempo de acceso (en min.) & 21,91 & 3,35 & 7 & 31 \\
\hline Bus público & \multicolumn{5}{|l|}{} \\
\hline Tiempo de espera (en min.) & 25,71 & 3,27 & 17 & 33 \\
\hline Bus público & 7,89 & 2,91 & 5 & 21 \\
\hline Costes del viaje $($ en $€$ ) & 14,9 & 1,17 & 9 & 20 \\
\hline Coche de alquiler & 5,87 & 1,25 & 4 & 6 \\
\hline Bus turístico & 4,16 & 0,67 & 3 & \\
\hline Bus público &
\end{tabular}

FUENTE: Elaboración propia. 
El Cuadro 5 muestra que, para llegar hasta el parque, el modo coche, tanto privado como de alquiler, es sustancialmente más rápido que el bus público o turístico. Concretamente, un 85 por 100 más rápido en promedio, contando además con una menor dispersión entre los encuestados. Con respecto a los costes del trayecto, el coche privado es el medio de transporte más competitivo, seguido de cerca por el bus público y por el coche de alquiler. En relación al coste del bus turístico, debe tenerse en cuenta que éste solo contempla el desplazamiento hasta el Teide y no otros servicios frecuentemente ofertados como la comida en algún restaurante. Finalmente, nótese que los tiempos de espera y acceso solo se dan para el autobús público ya que se asumió que no existen para el viaje en el autobús turístico (la recogida de los viajeros se produce en el propio hotel) y que el tiempo de acceso se refiere al desplazamiento a pie hasta la parada del autobús.

\section{Metodología}

En esta sección se presentan los fundamentos teóricos y se discuten brevemente los principales aspectos econométricos de los modelos de elección discreta utilizados en el análisis de la elección del modo de transporte por parte de los visitantes del Parque Nacional del Teide. El lector interesado puede encontrar una revisión extensiva de estos modelos en Train (2009), Hensher et al. (2015) y Ortúzar y Willumsen (2011).

Los modelos de elección discreta predicen la probabilidad de que un individuo elija una alternativa entre en un número finito de opciones discretas y excluyentes entre sí; en nuestro caso, entre distintos modos de transporte. Partiendo de la teoría de la utilidad aleatoria (Domencich y McFadden, 1975), es posible especificar una función indirecta de utilidad para cada modo de transporte, que representaría la máxima utilidad que un individuo puede obtener si elige esa alternativa. El individuo seleccionará la opción que le proporcione mayor utilidad en función de sus propias características y de las características del modo de transporte, por ejemplo, los tiempos en vehículo, de espera o los costes del desplazamiento. Debido a que no es posible observar todos los factores que afectan a las preferencias del individuo para discriminar entre una opción u otra, las funciones de utilidad han de especificarse como una variable estocástica incluyendo un componente de error aleatorio. Concretamente, la utilidad que un individuo $q$ asocia a un modo de transporte $j$ puede especificarse a través una función de utilidad:

$$
U_{j q}=V_{j q}\left(\beta_{j} x_{j q}\right)+\varepsilon_{j q}
$$

donde $V_{j q}$ es una función de utilidad indirecta y expresa el componente sistemático de la utilidad de la alternativa $j$, que es, a su vez, función de un vector $x_{j q}$ de características observables de la alternativa y del individuo, $\beta_{j}$ es un vector de coeficientes a estimar y es el componente de error aleatorio que refleja la parte no observable de la función de utilidad. 
Si la parte sistemática $V_{j q}$ de la función de utilidad [1] contiene atributos relativos al tiempo de viaje (T) y al coste del viaje (C) es posible calcular, mediante la ecuación [2], lo que se conoce como el valor subjetivo del tiempo de viaje (VSTV). Esta medida expresa la disposición a pagar de los individuos por ahorrar tiempo de viaje en un determinado modo de transporte. Si $V_{j q}$ es especificada de forma lineal, el VSTV es calculado simplemente mediante el ratio entre los coeficientes del tiempo y del coste de viaje,

$$
V S T V_{j q}=\frac{\frac{\partial V_{j q}}{\partial T_{j q}}}{\frac{\partial V_{j q}}{\partial C_{j q}}}
$$

La justificación de esta aproximación se sustenta en los modelos de asignación del tiempo (una revisión puede consultarse en González, 1997, y más recientemente en Jara-Díaz y Rosales-Salas, 2017). En estas teorías se asume que los individuos pueden obtener utilidad mediante el consumo de bienes y mediante el tiempo que ocupan en diversas actividades. Este comportamiento se representa a través de una función de utilidad directa que incluye bienes y tiempo, que trata de maximizarse sujeta a unas restricciones relativas a la cantidad de tiempo y de renta disponibles. En concreto, las especificaciones de los modelos de elección discreta que se estiman en este trabajo, y a partir de las cuales se obtienen los VSTV, se sustentan en el modelo de DeSerpa (1971), que sienta las bases para la construcción de la teoría microeconómica del valor del tiempo. En este modelo, el problema del consumidor puede plantearse como:

s.a.

$$
\max U\left(x_{1}, \ldots, x_{m} ; T_{1}, \ldots, T_{m} ; T_{w}\right)
$$

$$
\begin{array}{ll}
T_{w} \bar{w}+V \geq p_{i} x_{i} & {[\lambda]} \\
\sum T_{i}+T_{w} \leq T & {[\mu]} \\
T_{i} \geq a_{i} x_{i} \forall i & {\left[\psi_{i}\right]} \\
T_{w} \geq T_{w}^{0} & {[\phi]}
\end{array}
$$

donde la función de utilidad directa [3] depende del consumo de bienes $x_{i}$, un vector de tiempos $T_{i}$ que expresa la cantidad de tiempo asignada a cada bien y la cantidad de horas trabajadas $T_{w}$. Esta función está sujeta a una restricción presupuestaria [4], donde el consumo total de bienes $\left(p_{i} x_{i}\right)$ no puede exceder al total de ingresos salariales $\left(T_{w} \bar{w}\right)$ y no salariales $(V)$; una restricción de tiempo [5], donde la suma del tiempo dedicado al consumo de bienes y al trabajo no puede exceder el tiempo total disponible $(T)$; una restricción tecnológica [6], donde cada bien consumido requiere 
un tiempo mínimo proporcional a la cantidad consumida determinado por $a_{i}$, y una restricción [7] que asume que la cantidad de horas trabajadas no pueden exceder la duración de una jornada laboral $\left(T_{w}^{0}\right)$. Por su parte, $\lambda, \mu, \psi_{i}$ y $\phi$ son los multiplicadores de Lagrange asociados a cada una de las restricciones y se interpretan como la utilidad marginal del ingreso $(\lambda)$, del tiempo como recurso escaso $(\mu)$, de reducir el tiempo necesario para consumir un bien $\left(\psi_{i}\right)$ y de reducir en una unidad la cantidad de horas de trabajo $(\phi)$.

A partir de las condiciones de primer orden de este problema se obtienen distintas medidas del valor del tiempo. El cociente $\left(\partial U / \partial T_{i} / \lambda\right)$ es el valor del tiempo como mercancía y depende de si el tiempo genera utilidad o desutilidad cuando es consumido en una actividad, $\mu / \lambda$ es el valor del tiempo como recurso e indica el valor monetario de tener una unidad adicional de tiempo y $\psi_{i} / \lambda$ es el ratio marginal de sustitución entre el tiempo dedicado a una actividad y el dinero. Esta última expresión coincide con el concepto de VSTV expresarse como:

$$
\frac{\psi_{i}}{\lambda}=\frac{\mu}{\lambda}-\frac{\frac{\partial U}{\partial T_{i}}}{\lambda}
$$

En un contexto de demanda recreativa, como el de este trabajo, hay dos valores del tiempo relevantes. Por un lado, el tiempo que pasa el individuo en el espacio natural. En ese caso la restricción [6] no entra en juego, ya que el individuo dedica más tiempo del necesario a consumir el bien $\left(\psi_{i}=0\right)$ y, por tanto, el valor de ahorrar tiempo será 0 al no existir una alternativa que produzca mayor utilidad. Por otro lado, el tiempo que pasa el individuo en su desplazamiento hasta el parque. En este caso, ese tiempo puede ser ahorrado y transferido a otras actividades que generen mayor utilidad, por lo que su valor puede ser derivado a partir del trade-off que realizan los individuos a la hora de elegir entre un modo de transporte más rápido y caro o uno más lento y barato.

Una vez especificado correctamente el componente determinístico $V_{j q}$ de la función de utilidad [1], pueden estimarse distintos modelos de elección dependiendo del tratamiento de $V_{j q}$ y de $\varepsilon_{j q}$. En este trabajo se utilizan el modelo logit multinomial (MNL) y el modelo logit mixto (ML). La utilización del MNL constituye un buen punto de partida debido a sus ventajas en cuanto a facilidad de estimación, pero cuenta con dos grandes inconvenientes. Por un lado, asume que en [1] el vector de coeficientes $\beta_{j}$ es fijo en la población, no permitiendo fuentes de heterogeneidad aleatoria entre los individuos y, por otro lado, asume una distribución Gumbel independiente e idénticamente distribuida para vector de errores aleatorios $\varepsilon_{j q}$, lo que induce a la propiedad de independencia de alternativas irrelevantes. Una aproximación para superar estas limitaciones es introducir heterogeneidad en las preferencias a través de la interacción de los atributos del modo de transporte y las características socioeconómicas (CS) del individuo (véase, por ejemplo, Ortúzar y Willumsen, 2011, página 279). Asimismo, también se puede considerar una forma más general de 
heterogeneidad utilizando especificaciones ML y tratando a los coeficientes $\beta_{j}$ en [1] como variables aleatorias que siguen una distribución que depende de ciertos parámetros poblacionales, por ejemplo, la media y la desviación estándar. En este trabajo se utilizan ambas aproximaciones, por lo que la función de utilidad es especificada como:

$$
U_{j q}=V_{j q}\left(A S C_{j}+\beta_{j q} x_{j q} * C S_{q}\right)+\varepsilon_{j q}
$$

donde $\beta_{j q}$ es un vector de coeficientes aleatoriamente distribuido entre la población con media $\left(\beta_{j}\right)$ y desviación estándar $\left(\sigma_{j}\right)$ y $C S_{q}$ son características socioeconómicas del individuo. Para un determinado valor de $\beta_{j}^{q}$, la probabilidad condicional logit de elegir la alternativa $j$ por el individuo $q$ vendrá dada por:

$$
P_{i q}\left(\beta_{j}\right)=\frac{\exp \left(A S C_{j}+\beta_{j q} x_{j q} * C s_{q}\right)}{\sum_{i=1}^{I} \exp \left(A S C_{i}+\beta_{i q} x_{i q} * C S_{q}\right)}
$$

No obstante, dado que $\beta_{j}$ es desconocido, la probabilidad incondicional se calcula a partir de la integral de $P_{i q}\left(\beta_{j}\right)$ sobre todos los valores posibles que pueda tomar $\beta_{j}$ con los pesos dados por su densidad $f\left(\beta_{j} \mid \theta\right)$ :

$$
S P_{i q}=\int P_{i q}\left(\beta_{j}\right) f\left(\beta_{j} \mid \theta\right) d \beta
$$

Dado que esta integral no suele tener expresión analítica, esta debe ser aproximada numéricamente mediante simulación.

Por último, cabe comentar que el ML permite al analista especificar una determinada distribución para los parámetros $\beta_{j q}$ en [9]. Generalmente, las distribuciones más utilizadas son la normal, log-normal y triangular (Hess et al., 2005). No obstante, el cálculo de los valores subjetivos del tiempo de viaje cuando se consideran distintas distribuciones no es sencillo y puede conducir a interpretaciones erróneas de las disposiciones a pagar de los individuos. Por ejemplo, una distribución normal utilizada para el parámetro del coste del viaje podría implicar que cierto porcentaje de los individuos tenga una preferencia por precios más altos, lo cual contradice la lógica económica. No obstante, su aplicación no entrañaría grandes problemas cuando la probabilidad de obtener un signo erróneo para el parámetro es pequeña, dado que, aún considerando una distribución que no impone restricciones sobre el signo, se obtendrían resultados consistentes. Otras distribuciones, como la log-normal, permiten potencialmente resolver este problema, ya que es posible restringir el signo de la distribución. Sin embargo, su utilización puede presentar inconvenientes durante el proceso de estimación debido a que tienden a producir funciones de log-verosimilitud muy planas, dificultando la búsqueda de la solución óptima (véase el interesante estudio presentado en Sillano y Ortúzar, 2005). En este trabajo se emplean diversas distribuciones con objeto de comparar las posibles diferencias en la estimación de los valores del tiempo. 


\section{Resultados}

En esta sección se estudian los principales resultados de los modelos de elección discreta estimados (Cuadro 6) y de los valores del tiempo obtenidos (Cuadro 7) a partir de la información de la de las 751 encuestas de preferencias reveladas presentada en la sección 2.2. Como punto de partida, se estimaron modelos del tipo logit multinomial (MNL) para posteriormente estimar modelos logit mixto (ML) incorporando patrones más flexibles de heterogeneidad y correlación empleando la formulación presentada en la sección 3. Los modelos fueron estimados utilizando el software Python Biogeme (Bierlaire y Fetiarison, 2009) y 500 extracciones cuasi aleatorias.

Las variables explicativas utilizadas, cuyos estadísticos descriptivos se encuentran en la Cuadro 5, fueron las constantes específicas de cada alternativa (ASC), dejando como referencia la del modo coche de alquiler, los tiempos en vehículo $(T)$ y el coste $(C)$ para cada modo de transporte y los tiempos de acceso $(T A)$ y de espera (TE) exclusivamente para el modo de transporte bus público, así como interacciones con las características socioeconómicas $(C S)$ de los individuos (Cuadro 3). Específicamente, siguiendo la ecuación [9], se estimó una función indirecta de utilidad donde la utilidad del individuo $q$ asociada con el modo de transporte $j$ se expresa en la forma:

$$
V_{j q}=A S C_{j}+\beta_{j q} T_{j q} * C S_{q}+\delta T A q+\gamma T E_{q}+\alpha C_{j q}
$$

En un primer paso se probaron distintas especificaciones, mostrando en el Cuadro 6 los resultados de las estimaciones obtenidas con los mejores modelos. Se testaron parámetros específicos para cada alternativa para todas las variables utilizadas, pero los coeficientes de los tiempos en vehículo de los modos coche privado y de alquiler, por un lado, y de bus público y turístico, por otro, mostraron no ser significativamente distintos. El parámetro del coste de viaje no resultó significativamente distinto entre los cuatro modos de transporte disponibles, por lo que también fue especificado de forma genérica. Se probaron, también, diferentes estructuras anidadas mediante componentes de error (véase, por ejemplo, Walker et al., 2007) para analizar la correlación entre modos de transporte. Específicamente, se agruparon las alternativas coche privado y de alquiler y bus público y turístico, así como coche de alquiler y bus turístico frente al resto, para comprobar si las agrupaciones eran percibidas de forma similar por los individuos, pero ninguna de las especificaciones resultó significativa. La presencia de efecto renta también fue estudiado mediante la introducción en los modelos de la variable del coste elevada al cuadrado (Jara-Díaz y Videla, 1989), pero los resultados obtenidos indicaron que no existe un efecto renta significativo. Con respecto a la interacción de los atributos de los modos de transporte y las características socioeconómicas de los individuos, todas las variables fueron interaccionadas con dummies referidas al sexo (hombre $=1$ ), edad y nacionalidad del encuestado. Las interacciones con las distintas nacionalidades no resultaron 
significativas, pero si resultó la interacción de los tiempos en vehículo del coche de alquiler y privado con el sexo y la del bus turístico y público con el intervalo de menor edad de los visitantes (18-25 años). Por último, se especificaron parámetros aleatorios para todas las variables explicativas, pero solo se encontró heterogeneidad de preferencias aleatoria para los tiempos en vehículo del bus turístico y público. La interacción de este parámetro con la edad también fue significativa, indicando que parte de esa heterogeneidad viene explicada por la edad de los visitantes. Asimismo, para este parámetro se testó la distribución triangular, lognormal, uniforme y normal, resultando significativa esta última.

Las estimaciones mostradas en el Cuadro 6 indican que los coeficientes de los modelos MNL y ML presentan signos intuitivamente correctos y son significativamente distintos de cero con un nivel de confianza del 95 por 100, a excepción de las constantes específicas en ambos modelos y la desviación estándar del tiempo en vehículo así como la interacción de este atributo con la edad para las alternativas del bus, en el caso del modelo logit mixto. Las estimaciones también indican que el modelo ML cuenta con un mejor ajuste (el test de razón de verosimilitud entregó un valor $\mathrm{LR}=4,554$ superior al valor umbral $\chi_{1 g l, 95 \%}^{2}=3,84$ mejora la $\log$-verosimilitud) en relación al modelo MNL debido a tener en cuenta una mayor heterogeneidad de preferencias. Tanto en el MNL como en el ML, las interacciones significativas con el sexo y con la edad indican que, en el primer caso, los hombres y las mujeres perciben de forma distinta el tiempo en vehículo en coche de alquiler o privado y, en el segundo caso, que los individuos de entre 18 y 25 años perciben de forma diferente

\section{CUADRO 6}

MODELOS ESTIMADOS

\begin{tabular}{|l|c|c|c|c|}
\hline \multirow{2}{*}{ Variable } & \multicolumn{2}{c|}{ MNL } & \multicolumn{2}{c|}{ ML } \\
\cline { 2 - 5 } & Coeficiente & $t$ & Coeficiente & $t$ \\
\hline ASC bus tur. & $-2,55$ & $-1,08$ & $-3,38$ & $-0,87$ \\
\hline ASC bus púb. & 4,77 & 1,68 & 3,7 & 0,89 \\
\hline ASC coche priv. & 1,92 & 1,71 & 1,44 & 1,1 \\
\hline Tiempo en vehículo - Coche de alq. y priv. & $-0,0529$ & $-2,76$ & $-0,0917$ & $-2,17$ \\
\hline Tiempo en vehículo - Bus tur. y púb. & $-0,0299$ & $-3,83$ & $-0,066$ & $-2,11$ \\
\hline SD Tiempo en vehículo - Bus tur. y púb. & & & 0,0248 & 1,76 \\
\hline Tiempo de acceso - Bus púb. & $-0,194$ & $-3,13$ & $-0,214$ & $-3,18$ \\
\hline Tiempo de espera - Bus púb. & $-0,278$ & $-4,49$ & $-0,304$ & $-4,46$ \\
\hline Tiempo en vehículo - Coche de alq. y priv. $*$ Sexo & 0,0117 & 2,42 & 0,0189 & 2,52 \\
\hline Tiempo en vehículo - Bus tur. y púb. * Edad & 0,00894 & 5,97 & 0,0149 & 1,77 \\
\hline Coste & $-0,00258$ & $-6,8$ & $-0,00397$ & $-2,83$ \\
\hline log-verosimilitud & \multicolumn{2}{|c|}{-297.572} & & -295.295 \\
\hline
\end{tabular}

FUENTE: Elaboración propia. 
el tiempo en vehículo en bus turístico o público con respecto a los individuos de mayor edad. Nótese, también, que el ML incluye la desviación estándar relativa al parámetro del tiempo en vehículo en bus turístico y público y su estadístico $t$ asociado, indicando que el parámetro aleatorio con distribución normal es significativo. A este respecto, cabe señalar que la variabilidad en las preferencias inducida por la distribución normal da como resultado que la probabilidad de obtener un signo erróneo (positivo) para este coeficiente es muy baja $(0,0039)$, lo cual hace que la interpretación de la utilidad marginal del tiempo en el vehículo para el bus sea consistente desde el punto de vista económico.

Por su parte, el Cuadro 7 muestra las estimaciones puntuales de los valores subjetivos del tiempo de viaje obtenidos a partir de las estimaciones del Cuadro 6. Como se especificó en la sección de metodología, estos valores del tiempo se obtienen a partir del ratio entre las utilidades marginales del tiempo (en vehículo, de espera y de acceso) y la utilidad marginal del dinero (coste del viaje).

Lo primero que podemos resaltar del Cuadro 7 es que los modelos MNL subestiman los VSTV en relación a los ML en todos los casos salvo en los tiempos de acceso y espera en bus público. Generalmente, es más usual encontrar en la literatura subestimaciones del modelo logit multinomial en relación al logit mixto (Amador et al., 2005; Hess et al., 2005; González et al., 2016), aunque también se observa la situación contraria (por ejemplo, Espino et al., 2008). Estas diferencias pueden ser explicadas por la inclusión de mayor heterogeneidad en los modelos ML, como en nuestro caso, la forma funcional elegida para la función de utilidad o por las peculiaridades de los datos utilizados (Sillano y Ortúzar, 2005). Asimismo, también es de resaltar que todos los valores del tiempo obtenidos tienen una magnitud superior a la aproximación habitual (Cesario, 1976) de un tercio de la tasa salarial (los datos para el cálculo de esta tasa pueden consultarse en el Apéndice). De hecho, tomando como referencia los valores del tiempo en vehículo, se observa como los valores del tiempo oscilan entre un 43 por 100 y un 123 por 100 de la tasa salarial.

Otro resultado a destacar, tomando como referencia nuestro mejor modelo (ML), son las diferentes magnitudes de los valores del tiempo de los visitantes del Parque Nacional del Teide. La percepción del tiempo de un visitante vendrá determinada por la desutilidad que le produce el tiempo empleado en el viaje y por el coste de oportunidad de asignar ese tiempo a otras actividades, por lo que es esperable que el valor del tiempo varíe de acuerdo a las condiciones en las que se realiza el viaje, a las características del modo de transporte y a las propias características del individuo. En efecto, analizando los resultados del Cuadro 7 se comprueba como los tiempos de espera y de acceso del bus público son los que presentan valores más elevados, especialmente el de espera que es el que provoca mayor desutilidad entre los visitantes. Este resultado es común encontrarlo en la literatura donde generalmente los valores del tiempo de espera duplican a los valores del tiempo en vehículo (Wardman, 2004). Asimismo, el valor del tiempo en los modos de transporte coche de alquiler y privado es ligeramente superior a los modos de transporte en bus. Una posible explicación podría ser que el viaje en el bus es más confortable y ofrece más 


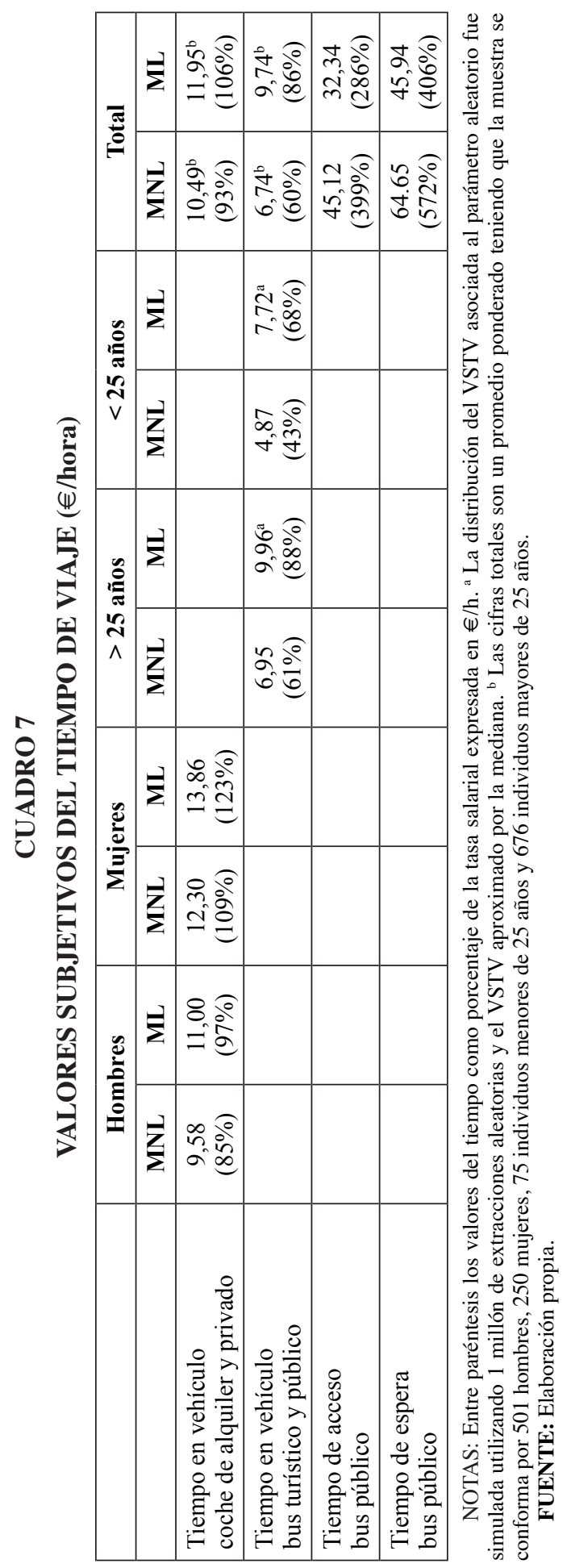


oportunidades de hacer un uso productivo del tiempo al liberar al viajero de conducir o de comprobar que la ruta hasta el parque es la correcta. Por último, destacan las diferentes magnitudes de los VSTV según el sexo y la edad del visitante. Se observa como los hombres otorgan una menor valoración del tiempo en vehículo en coche de alquiler o privado en relación a las mujeres y que los visitantes de entre 18 y 25 años tienen una disposición a pagar por ahorros de tiempo en bus público o turístico menor a la de resto de visitantes. Esto último podría explicarse por una mayor predisposición entre los jóvenes a utilizar medios de transporte públicos o colectivos para llegar hasta el parque en relación al modo de transporte coche, independientemente de la duración del viaje.

\section{Conclusiones}

En este trabajo se han obtenido estimaciones del valor del tiempo de viaje de los visitantes del Parque Nacional del Teide en sus desplazamientos hasta dicho parque. Para ello, partiendo de los fundamentos teóricos del modelo de asignación del tiempo de DeSerpa (1971), se han estimado diversos modelos de elección discreta referidos a la elección del modo de transporte.

En el análisis realizado se ha puesto de manifiesto la existencia de heterogeneidad en las preferencias de los visitantes turistas cuando eligen su modo de transporte. Dicha heterogeneidad se traslada a las disposiciones a pagar por ahorrar tiempo en las distintas etapas del viaje y viene explicada, fundamentalmente, por características socioeconómicas como el género y la edad y por otros factores de naturaleza aleatoria.

Asimismo, se ha mostrado que los diversos componentes del tiempo de viaje son valorados de forma distinta por los visitantes. Así, las disposiciones a pagar por reducir los tiempos de acceso y espera en el servicio de transporte público son sustancialmente más elevadas que las obtenidas por ahorrar tiempo de viaje en el vehículo.

Finalmente, los resultados obtenidos en este trabajo proporcionan instrumentos de gran utilidad a la hora de evaluar políticas que promuevan la utilización de modos de transporte más sostenibles y eficientes para el acceso a espacios naturales. Dichas políticas deben ser consideradas como prioritarias en espacios como el Parque Nacional del Teide, cuya capacidad de carga se encuentra comprometida en determinados momentos del día y espacios puntuales debido al gran volumen de visitantes que recibe. 


\section{Referencias bibliográficas}

[1] AMADOR, F. J.; GONZÁLEZ, R. M. y ORTÚZAR, J. d. D. (2005). «Preference heterogeneity and willingness to pay for travel time savings». Transportation, 32, 627-647.

[2] ANSSON, R. (1998). «Our national parks-overcrowded, underfunded, and besieged with a myriad of vexing problems: how can we best fund our imperiled national park system?». Journal of Land Use \& Environmental Law, 14 (1), 1-52.

[3] BALMFORD, A.; BERESFORD, J.; GREEN, J.; NAIDOO, R.; WALPOLE, M. y MANICA, A. (2009). «A global perspective on trends in nature-based tourism». PLoS biology, 7 (6), e1000144.

[4] BECKER, G. S. (1965). «A Theory of the Allocation of Time». The Economic Journal, 493-517.

[5] BIERLAIRE, M. y FETIARISON, M. (2009). Estimation of discrete choice models: extending BIOGEME, Workshop on discrete choice models, Ecole Polytechnique Fédérale de Lausanne, Lausanne, Switzerland.

[6] BUCKLEY, R. (2000). «Neat trends: Current issues in nature, eco-and adventure tourism». The International Journal of Tourism Research, 2 (6), 437.

[7] CESARIO, F. J. (1976). «Value of time in recreation benefit studies». Land Economics, $52(1), 32-41$.

[8] DeSERPA, A. C. (1971). «A theory of the economics of time». The Economic Journal, 81 (324), 828-846.

[9] DOMENCICH, T. y McFADDEN, D. (1975). «Urban Travel Demand: A Behavioral Analysis». North-Holland, Amsterdam.

[10] ESPINO, R.; MARTÍN, J. C. y ROMÁN, C. (2008). «Analyzing the effect of preference heterogeneity on willingness to pay for improving service quality in an airline choice context. Transportation Research Part E». Logistics and Transportation Review, 44 (4), 593-606.

[11] GONZÁLEZ, R. M. (1997). «The value of time: a theoretical review». Transport Reviews, 17, 245-266.

[12] GONZÁLEZ, R. M.; MARRERO, Á. S. y MARRERO, G. A. (2016). «How the values of travel time change when a panel data around a new tram implementation is used». European Journal of Transport \& Infrastructure Research, 16 (4).

[13] GÜRLÜK, S. y REHBER, E. (2008). «A travel cost study to estimate recreational value for a bird refuge at Lake Manyas, Turkey». Journal of Environmental Management, 88 (4), 1350-1360.

[14] HAGERTY, D. y MOELTNER, K. (2005). «Specification of driving costs in models of recreation demand». Land Economics, 81 (1), 127-143.

[15] HENSHER, D.A.; ROSE, J. M. y GREENE, W. H. (2015). Applied choice analysis. 2nd edition. Cambridge University Press, Cambridge.

[16] HESSS, S.; BIERLAIRE, M. y POLAK, J. (2005). «Estimation of value of travel-time savings using mixed logit models». Transportation Research: Part A. Policy and Practice, 39 (2-3), 221-236.

[17] JARA-DÍAZ, S. y ROSALES-SALAS, J. (2017). «Beyond transport time: A review of time use modeling». Transportation Research: Part A. Policy and Practice, 97, 209-230.

[18] JARA-DÍAZ, S. R. y VIDELA, J. (1989). «Detection of income effect in mode choice: Theory and application». Transportation Research Part B: Methodological, 23, 393-400. 
[19] MACE, B. L.; BELL, P. A. y LOOMIS, R. J. (2004). «Visibility and natural quiet in national parks and wilderness areas: Psychological considerations». Environment and Behavior, 36 (1), 5-31.

[20] MANNING, E. W. y DOUGHERTY, T. D. (2000). «Planning sustainable tourism destinations». Tourism Recreation Research, 25 (2), 3-14.

[21] MANNING, R. E.; LAUSON, S.; NEWMAN, P.; Hallo, J. y MONZ, C. (2014). Sustainable transportation in the National Parks, University Press of New England, Hanover and London.

[22] ORTÚZAR, J. DE D. y WILLUMSEN, L. G. (2011). Modelling Transport. 4th edition, John Wiley \& Sons, Chichester.

[23] PALMQUIST, R. B.; PHANEUF, D. J. y SMITH, V. K. (2010). «Short run constraints and the increasing marginal value of time in recreation». Environmental and Resource Economics, 46 (1), 19-41.

[24] REEVES, R. (2006). Tackling traffic: sustainable leisure transportation in national parks-An overview of national park authority involvement. Report by the Council of National Parks, 1-41.

[25] ROYSTON, P. y WHITE, I. R. (2011). «Multiple imputation by chained equations (MICE): implementation in Stata». Journal of Statistical Software, 45 (4), 1-20.

[26] SILLANO, M. y ORTÚZAR, J. DE D. (2005). «Willingness-to-pay estimation with mixed logit models: some new evidence». Environment and Planning, 37A, 525-550.

[27] TRAGSATEC (2015). Informe de visitantes del Parque Nacional del Teide. Informe realizado para el Parque Nacional del Teide.

[28] TRAIN, K. (2009). Discrete Choice Methods with Simulation, 2nd edition, Cambridge University Press, Cambridge.

[29] UNESCO (2007). «Proposal to inscribe Teide National Park on the world heritage list». Nomination file submitted to the World Heritage Center in January 2006 by the Spain Ministry of Environment and the Canary Government.

[30] WALKER, J. L.; BEN-AKIVA, M. y BOLDUC, D. (2007). «Identification of parameters in normal error component logit-mixture (NECLM) models». Journal of Applied Econometrics, 22 (6), 1095-1125.

[31] WARDMAN, M. (2004). «Public transport values of time». Transport Policy, 11 (4), 363-377. 


\section{APÉNDICE}

Dado que no se cuenta con información sobre el ingreso de los encuestados, la tasa salarial debe estimarse a partir de otras fuentes de información. Según las autoridades del parque, los visitantes por nacionalidad se distribuyen de la forma especificada en el Cuadro A.1.

CUADRO A.1

VISITANTES DEL PARQUE NACIONAL DEL TEIDE SEGÚN NACIONALIDAD (AÑ 2015)

\begin{tabular}{|l|c|}
\hline \multicolumn{1}{|c|}{ Nacionalidad } & Porcentaje de visitas \\
\hline Española & 25,86 \\
\hline Alemana & 11,37 \\
\hline Francesa & 9,91 \\
\hline Británica & 9,26 \\
\hline Italiana & 5,17 \\
\hline Resto del mundo & 38,43 \\
\hline
\end{tabular}

FUENTE: Elaboración propia.

En el Cuadro A.2 se presenta la información relativa al nivel de ingreso y horas trabajadas de las nacionalidades especificadas en el Cuadro A.1. La tasa salarial es calculada como la ratio entre el ingreso anual personal y el promedio anual de horas trabajadas para cada nacionalidad. La media ponderada es calculada según el peso que representa cada nacionalidad sobre el total de visitantes, especificados en el Cuadro A.1.

\section{CUADRO A.2}

INGRESO Y HORAS TRABAJADAS POR NACIONALIDAD

\begin{tabular}{|c|c|c|c|}
\hline Nacionalidad & $\begin{array}{c}\text { Ingreso total } \\
\text { disponible anual } \\
(€)(\mathbf{a})\end{array}$ & $\begin{array}{c}\text { Horas trabajadas } \\
\text { anuales } \\
(\mathbf{b})\end{array}$ & $\begin{array}{c}\text { Tasa salarial } \\
(\mathbf{a} / \mathbf{b})(€ / \mathbf{h})\end{array}$ \\
\hline Española & 13.681 & 1.695 & 8,07 \\
\hline Alemana & 21.275 & 1.363 & 15,61 \\
\hline Francesa & 21.713 & 1.472 & 14,75 \\
\hline Británica & 21.136 & 1.676 & 12,61 \\
\hline Italiana & 15.846 & 1.730 & 9,16 \\
\hline Media ponderada & 17.679 & 1.598 & 11,31 \\
\hline
\end{tabular}

FUENTE: Eurostat, «Total disposable income, 2015», y OCDE, «Average annual hours actually worked per worker, 2015». 